\title{
Non-COVID-19 emergencies: where have all the patients gone?
}

\author{
Tom Boeken $^{1} \cdot$ Alice Le Berre $^{1} \cdot$ Anne Mebazaa $^{1} \cdot$ Isabelle Boulay-Coletta ${ }^{1} \cdot$ Jérôme Hodel $^{1} \cdot$ Marc Zins $^{1}$ (D)
}

Received: 7 May 2020 / Accepted: 15 May 2020 / Published online: 26 May 2020

(C) European Society of Radiology 2020

\section{Key Points}

- Early in the pandemic, numbers of patients undergoing non-COVID-19 emergent CTs dropped sharply but diagnostic yield did not increase, suggesting potentially undiagnosed emergencies in patients not seen in healthcare institutions.

As COVID-19 cases exploded in Europe in March and April 2020 [1], the number of patients seen in emergency departments surged, and radiology departments reallocated resources to serve the sudden influx of patients requiring chest $\mathrm{CT}$ for suspected or confirmed COVID-19 [2, 3]. Simultaneously, the number of non-COVID-related CTs dramatically dropped [4], questioning if true non-COVID-19 case illnesses were correctly managed.

The objective of this study was to assess if the pandemic had modified the profile of CT results in patients with nonCOVID-19 medical emergencies in our institution. In other words, we aimed to evaluate which "non-COVID" patients refrained from undergoing $\mathrm{CT}$ during the pandemic.

This retrospective study was approved by our institutional review board. Non-COVID emergency department patients who underwent CT during March 16-30, 2019 (control group), and March 16-30, 2020 (study group), were included. The lockdown was officially pronounced on March 17, 2020. There were no exclusion criteria.

Emergency department and radiology files were evaluated by two radiologists, by consensus. CT scans were categorized as positive when the radiological diagnosis explained the clinical findings or negative in other cases (normal or unrelated findings).

The dataset consisted of 416 CTs in the 2019 control period and 465 CTs in the 2020 study period. During the study period, 301 CTs were performed for COVID-related emergencies and 164 CTs for non-COVID emergencies corresponding to a drop of $61 \%$. Mean age (years) was $62 \pm 24$ and the female/ male ratio was $51 / 49$, similar for the two periods.

Marc Zins

mzins@hpsj.fr

1 Department of Radiology, Groupe Hospitalier Paris Saint-Joseph, 185 rue Raymond Losserand, 75014 Paris, France
CT scans for non-COVID emergencies were positive in 132 controls (32\%) in 2019 and in 59 patients (36\%) during the same period in 2020 ( -73 patients). In-depth analysis showed no statistically significant difference between the groups in both the anatomic profile of CTs performed and the exam yield for non-COVID patients.

This study shows a sharp drop in non-COVID-19-related CTs during the current health crisis compared with a similar period in 2019. One hypothesis would have been that patients with mild symptoms or minor disease would refrain from going to emergency department, while those with more worrying conditions would have maintained their visit. However, this is not the case. The proportion of positive CTs did not increase compared with that in 2019. Therefore, the decrease in the number of CTs in standard-track patients is more likely a consequence of patient unwillingness to attend emergency departments, due to fear of contracting COVID-19. This means that the diagnosis of potentially severe conditions might not be done or at least significantly delayed. This is of major concern and specific public health actions may be needed.

Funding information The authors state that this work has not received any funding.

\section{Compliance with ethical standards}

Guarantor The scientific guarantor of this publication is Marc Zins, MD.

Conflict of interest The authors of this manuscript declare no relationships with any companies whose products or services may be related to the subject matter of the article.

Statistics and biometry No complex statistical methods were necessary for this paper. 
Informed consent Written informed consent was waived by the Institutional Review Board.

Ethical approval Institutional Review Board approval was obtained.

\section{Methodology}

- retrospective

- observational

- performed at one institution

\section{References}

1. European Centre for Disease Prevention and Control (2020) Situation update worldwide, as of 6 April 2020. https://www.ecdc. europa.eu/en/geographical-distribution-2019-ncov-cases. Accessed 17 Apr 2020

2. Politi LS, Balzarini L (2020) The radiology department during the COVID-19 pandemic: a challenging, radical change. Eur Radiol. https://doi.org/10.1007/s00330-020-06871-0

3. Revel MP, Parkar AP, Prosch H et al (2020) COVID-19 patients and the radiology department - advice from the European Society of Radiology (ESR) and the European Society of Thoracic Imaging (ESTI). Eur Radiol. https://doi.org/10.1007/s00330-020-06865-y

4. Cavallo JJ, Forman HP (2020) The economic impact of the COVID19 pandemic on radiology practices. Radiology. https://doi.org/10. 1148/radiol.2020201495:201495

Publisher's note Springer Nature remains neutral with regard to jurisdictional claims in published maps and institutional affiliations. 